\title{
Internado rural en la carrera de medicina de la Universidad de Chile; una experiencia de aprendizaje significativo.
}

\author{
Rural Clerkship in Medical Studies of the University of Chile: \\ an experience of significative learning
}

\begin{abstract}
Teresa Millán $\mathrm{K}^{1}$, N. A. Vargas $\mathrm{C}^{2}$. Nelly Madrid $\mathrm{C}^{3}$.
Departamento de Pediatría y Cirugía Infantil, Facultad de Medicina, Campus Occidente, Universidad de Chile., Corporación Municipal para la Educación y Salud de Melipilla ${ }^{3}$.
\end{abstract}

El Internado de la Carrera de Medicina constituye la etapa final en la formación del médico que realiza su estudios en la Facultad de Medicina de la Universidad de Chile, Santiago de Chile. Su objetivo principal, en los dos años que lo conforman, es que el alumno logre poner en práctica los conocimientos recibidos, adquiriendo y perfeccionando las competencias para el enfrentamiento práctico de problemas clínicos.

El Internado Rural, que queremos difundir en esta colaboración, es una experiencia obligatoria que deben cursar los alumnos en el $7^{\circ}$ año (último) de la carrera. Existen, además, Internados de Medicina, Cirugía, Obstetricia y Especialidades, todos ellos en el último año de la formación curricular. El internado rural es una experiencia integradora de la enseñanza de la Salud Pública en terreno y su conexión con las disciplinas clínicas, conduce a la consolidación de la autonomía del futuro médico .

El estudiante se incorpora al medio rural, apreciando las condiciones de vida y las características de la atención de salud. Se pone especial énfasis en

\footnotetext{
Correspondencia:

Teresa Millán

Las Palmeras 299 Interior

Quinta Normal Casilla 33052 Correo 33

Santiago Chile.

e-mail:tmillan@med.uchile.cl
}

las acciones de promoción, fomento, prevención, recuperación, rehabilitación y reinserción social, a lo largo de todas las etapas del ciclo vital individual y familiar acentuando el enfoque biopsicosocial. Al mismo tiempo, la relación con profesionales, trabajadores de la salud y organizaciones comunitarias brinda la oportunidad de desarrollar habilidades de liderazgo y trabajo en equipo, fundamentales para su ejercicio profesional.

A lo largo del tiempo el programa permite llevar a cabo proyectos que, en el mediano y largo plazo, contribuyen a mejorar el nivel de salud de la comunidad, reflejando de esta manera el compromiso de la Universidad de Chile con la población. Esta posibilidad permite, adicionalmente, que los internos se sientan parte de un esfuerzo mantenido en el tiempo y no de acciones esporádicas.

Esta experiencia es una tradición consolidada por más de treinta años y la han recibido los internos en diversas localidades del área rural que se describe más adelante. El lugar más estable ha sido San Manuel, en la Comuna de Melipilla, localidad rural dispersa situada a 90 kilómetros de la ciudad capital de Chile, Santiago.

Los objetivos que plantea el programa se cumplen con un conjunto de actividades prácticas que permiten fortalecer y desarrollar habilidades en promoción de salud (creación, planificación, desarrollo y evaluación de programas y proyectos en salud), atención primaria de salud y relaciones humanas con una comunidad rural.

La metodología aplicada para el proceso de enseñanza aprendizaje se basa en el enfoque constructi- 
vista $\mathrm{y}$ es supervisada por profesores tutores $\mathrm{y}$ encargado del programa por parte de la Universidad de Chile, coparticipando un tutor local del Departamento de Salud del gobierno local (Corporación de Salud y Educación de Melipilla).

El internado consiste en la inserción por cuatro semanas de dos grupos de internos, desde enero a diciembre. Ellos tienen residencia permanente en los consultorios y postas rurales "El Bollenar" y "San Manuel", dependientes de la corporación mencionada. Gozan de libertad para el diseño, ejecución y evaluación de actividades en promoción de salud y atención primaria en la comunidad. Se programan y ejecutan 12 programas, integrados en forma intersectorial y en red, con el equipo de salud local y la comunidad organizada (Comité Local de Salud, Jardines Infantiles, Colegios y Liceos, grupos de pacientes crónicos, trabajadoras temporeras).

La planificación anual de proyectos y programas tiene concordancia con los programas institucionales y permite ejecutar; así como evaluar actividades de promoción y educación en salud para grupos específicos de niños/as, adolescentes, mujeres jóvenes y adultos/as mayores. Además, se brinda atención primaria para: a) detectar trastornos oftalmo- lógicos y osteomusculares; b) diagnóstico nutricional; c) atención domiciliaria a pacientes postrados; d) promoción de salud dental; e) detección de problemas de salud prevalentes y f) labor asistencial en los consultorios y postas con un total de 200 atenciones promedio mensuales por cada grupo de internos.

La evaluación del proceso docente; así como el conjunto de la experiencia se puede objetivar en evaluaciones sucesivas que cada grupo realiza al término de su período. Las ideas que arrojan estas evaluaciones ponen énfasis en que este internado, más que actividad docente-asistencial, es una instancia de gran aprendizaje y una experiencia de vida. Los internos declaran que otorga destrezas para enfrentar y resolver problemas de salud familiar y comunitaria, y afirman que es igualmente beneficioso para la población. Por otra parte, es un primer contacto directo, autónomo con una realidad social no vista antes en el transcurso de su carrera. En esta experiencia el interno actúa en forma independiente, con supervisión indirecta, poniendo en práctica todo el acervo académico adquirido. Ello permite una expansión de la creatividad, no explorada antes debido a la tendencia de programas rígidos de educación. 